University of Louisville ThinkIR: The University of Louisville's Institutional Repository

Electronic Theses and Dissertations

$4-2018$

\title{
Tumor-targeted double emulsions for ultrasound- triggered delivery of molecular therapeutics.
}

Connor S Centner

University of Louisville

Follow this and additional works at: https://ir.library.louisville.edu/etd

Part of the Biological Engineering Commons

\section{Recommended Citation}

Centner, Connor S, "Tumor-targeted double emulsions for ultrasound-triggered delivery of molecular therapeutics." (2018). Electronic Theses and Dissertations. Paper 3002.

https://doi.org/10.18297/etd/3002

This Master's Thesis is brought to you for free and open access by ThinkIR: The University of Louisville's Institutional Repository. It has been accepted for inclusion in Electronic Theses and Dissertations by an authorized administrator of ThinkIR: The University of Louisville's Institutional Repository. This title appears here courtesy of the author, who has retained all other copyrights. For more information, please contact thinkir@louisville.edu. 


\title{
By
}

Connor Sterling Centner

B.S., University of Louisville, 2017

\author{
A Thesis \\ Submitted to the Faculty of the \\ University of Louisville \\ J.B. Speed School of Engineering \\ As Partial Fulfillment of the Requirements \\ For the Professional Degree
}

\section{MASTER OF ENGINEEERING}

Department of Bioengineering

May 2018 

TUMOR-TARGETED DOUBLE EMULSIONS FOR ULTRASOUND-TRIGGERED DELIVERY OF MOLECULAR THERAPEUTICS

Submitted by:

Connor Sterling Centner

A Thesis Approved On

(Date)

By the Following Reading and Examination Committee:

Jonathan A. Kopechek, Ph.D, Thesis Director

Martin G. O’Toole, Ph.D

Guruprasad A. Giridharan, Ph.D

Paula J. Bates, Ph.D 


\begin{abstract}
Cancer is the second leading cause of death in the United States, with 1.74 million new cancer cases diagnosed and 610,000 cancer deaths expected in 2018 alone. Current treatments often result in negative systemic effects and ineffective treatment of the tumor. Drug delivery vehicles have been developed for more effective local delivery methods, but many drug delivery vehicles lack spatial and temporal control. Targeted double emulsions are a new class of drug delivery vehicles which present a promising option for a high payload and controlled delivery. The purpose of our project was to develop and characterize an aptamer-chelated double emulsion that has the ability to actively target cancer cells and can be activated with ultrasound. AS1411, a 26-base guanine-rich oligonucleotide (GRO), was selected since AS1411 has the ability to target nucleolin surface receptors, which are overexpressed in many cancerous cell lines. Perfluorohexane forms the shell of the drug delivery vehicle since it is ultrasound-responsive in clinically acceptable pressure ranges. Ultrasound applied to double emulsions will cause vaporization of the perfluorocarbon shell, allowing transport of the molecular compound into the cancer cell with a higher efficiency. If a higher concentration is inside the cell than the microenvironment, the transient pores can release the molecular compound from the cytoplasm. This was seen in our static condition $(\mathrm{p}=0.054)$. A fluidic model is needed since static conditions doesn't accurate depict conditions that will be seen in vivo. The payload was released from core of the double emulsion. To help reduce the passive release of the payload, different surfactants were testing. FluorN562 showed a slower release profile than FluorN561 and Poloxamer 188 at $4{ }^{\circ} \mathrm{C}, 21{ }^{\circ} \mathrm{C}$, and $37{ }^{\circ} \mathrm{C}(\mathrm{p}<0.05)$, with $21{ }^{\circ} \mathrm{C}$ being the optimal temperature for storage of double emulsions $(\mathrm{p}<0.01)$. Polydispersed double
\end{abstract}


emulsions varied too greatly in size for the double emulsions to be used clinically. To address this issue, a microfluidic device was developed for the creation of monodispersed double emulsions under 10 microns. Double emulsions, once steady state steady flow was reached, were constantly between 1 to $2 \mu \mathrm{m}$. Ultrasound-responsive double emulsions could a potential significant impact clinically allowing for therapeutic molecular compounds to enter the cancer cell more easily through the transient pores, which is a distinct advantage to this drug delivery system. 


\section{TABLE OF CONTENTS}

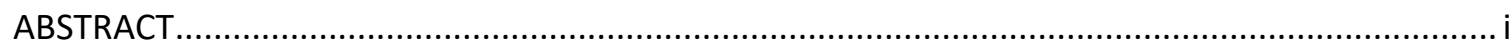

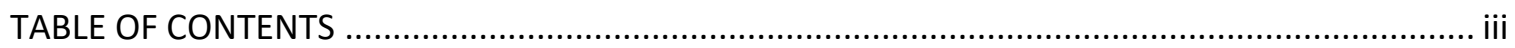

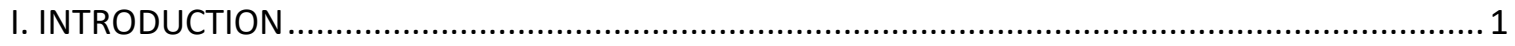

II. Targeted Drug Delivery to Cancer Cells in Static Culture Conditions ....................................... 5

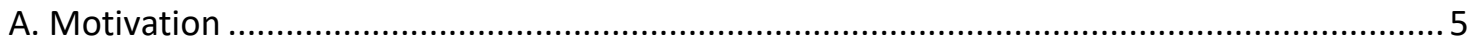

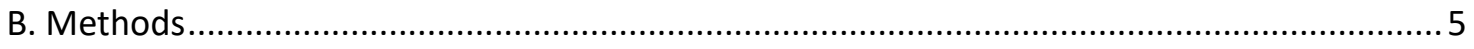

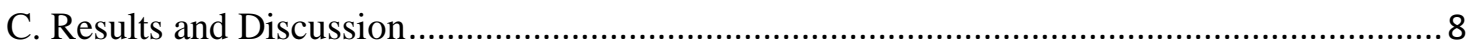

III. Characterization of Surfactant Effects on Double Emulsion Stability ..................................... 14

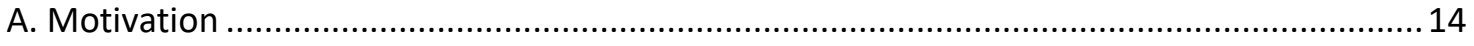

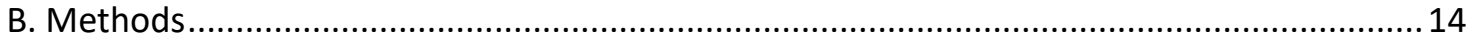

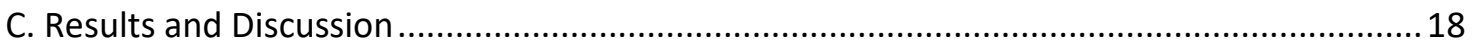

IV. Microfluidic Synthesis of Monodisperse Double Emulsions................................................22

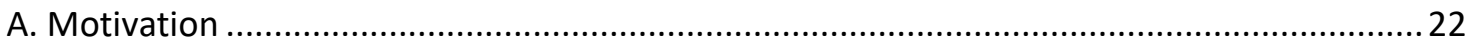

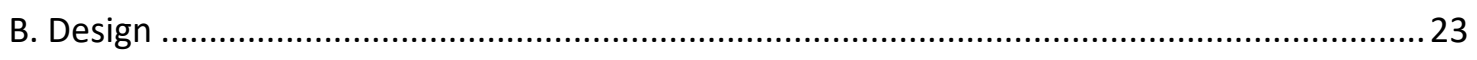

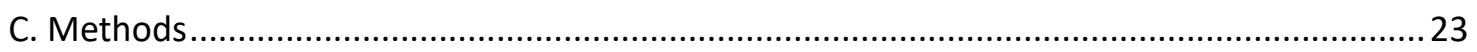

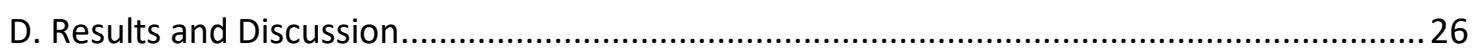

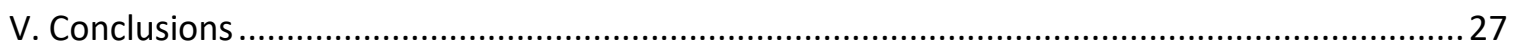

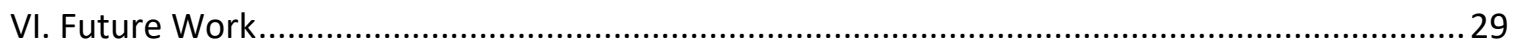

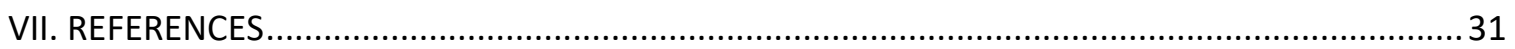




\section{INTRODUCTION}

In the United States, cancer is the second leading cause of death with an estimated 610,000 deaths in 2018. While advances in diagnostics and treatment methods have occurred, 5 year survival rate is $67 \%$ according to Surveillance, epidemiology, and End Results [SEER] program. Advances in treatment methods are necessary to increase survival rates in the cancer population. Many treatment options are possible for the treatment of cancer, but chemotherapy still remains one of the most commonly used options for treatment. Chemotherapy treatment has high systemic toxicity and lacks effective local delivery to tumors.[1,2] Multiple reasons can explain the lack of effective local delivery, including lack of specificity and rapid clearance in circulation.

To help address these issues, drug delivery vehicles have been developed to improve specificity and reduce systemic toxicity of chemotherapy.[3] Antibodies and other targeting compounds such as aptamers have been added to drug delivery vehicles to improve specificity.[3, 4] However, many drug delivery vehicles still lack in effectiveness since the drug delivery vehicles often do not efficiently get the chemotherapy into the cancer cell.[5-7]

To address this limitation, targeted, ultrasound-responsive double emulsions have been synthesized to improve the effectiveness of delivering hydrophilic molecular compounds to the cell of interest. [8-10] Historically, double emulsions have been used for over a hundred years in food and cosmetics industries, but limited research has been conducted for biomedical use.[11] Emulsions consist of two immiscible liquids, in which one liquid is dispersed within the other causing a droplet to form. A major limitation of double emulsions is that double emulsions tend to coalesce without an effective 
surfactant.[12] However, recent developments in synthesis of new biocompatible surfactants has helped improve the stability of double emulsions allowing further research to be conducted.[13-15] However, lack of an effective controlled release methodology has hindered translation to clinical use. To address this limitation, targeted, ultrasoundresponsive formulated double emulsions have been developed to enable spatial and temporal control over release of encapsulated
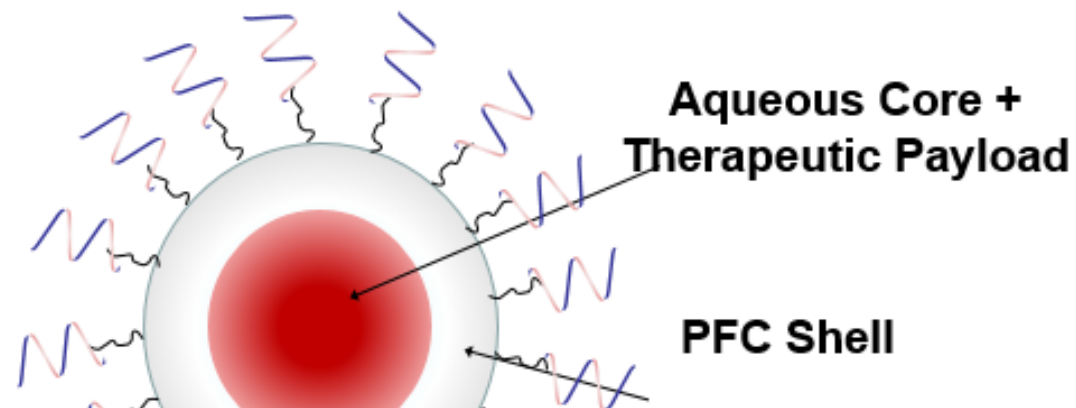

compounds.[16] Ultrasound-responsive double emulsions are composed of a liquid perfluorohexane middle phase encapsulating an aqueous inner phase, shown in figure 1. Perfluorohexane is used as the middle phase to prevent hydrophilic drugs from entering the bloodstream and diffusing into any cell type. Perfluorohexane is chemically and biologically inert with a low boiling point, which allows it to be vaporized into gas phase with ultrasound pulses.

Ultrasound has been widely used for decades in medical and engineering applications, including non-destructive testing of materials. More recently, ultrasound applications in therapeutics have been investigated. Studies have demonstrated ultrasoundenhanced therapeutic delivery to cells in vitro and in vivo. $[8,9,14]$ 


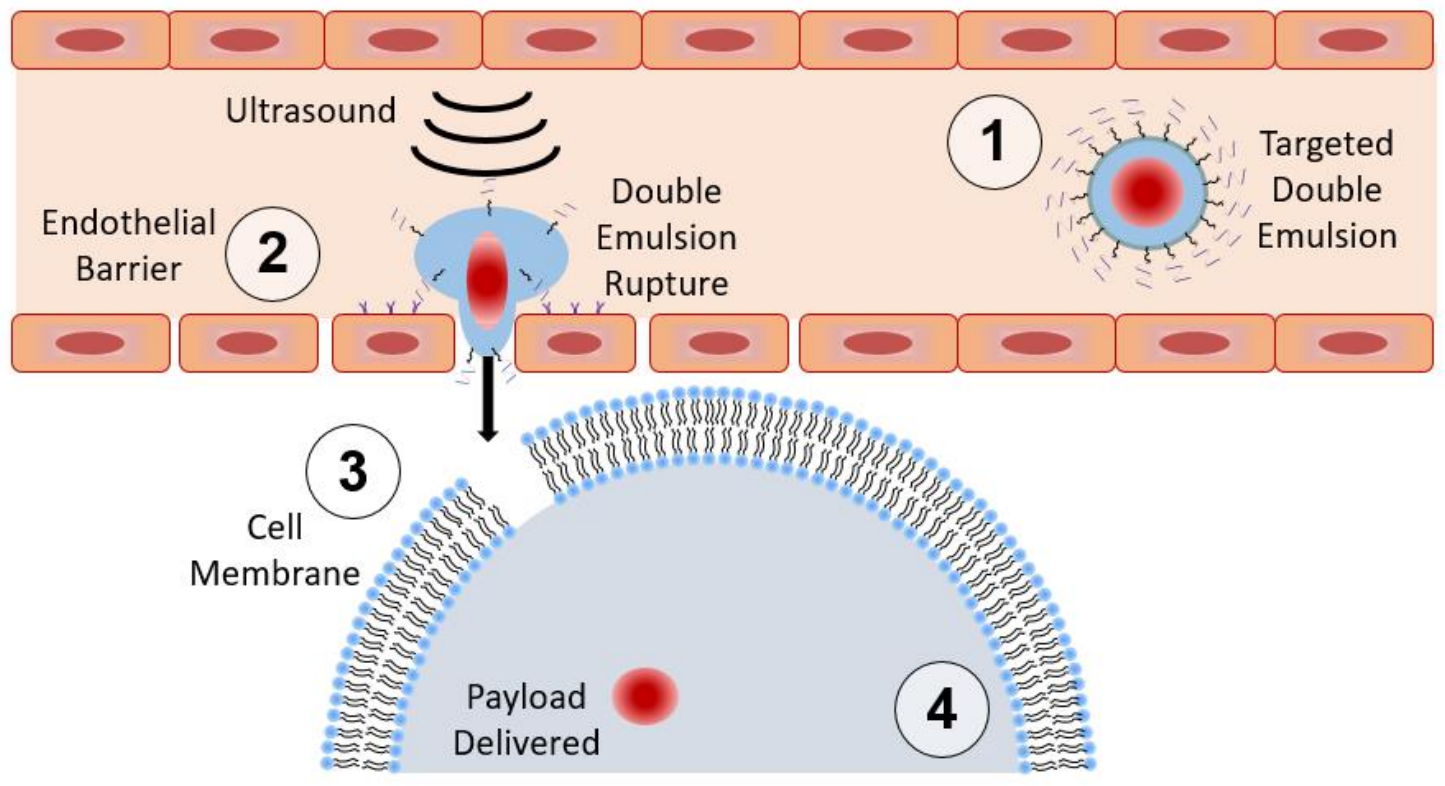

Figure 2- Schematic of inertial cavitation induced by vaporization of double emulsion, which causes transient pores in the cell membrane.

This delivery technique generally facilitates transmembrane transport of drug by causing oscillatory behavior of double emulsions in response to ultrasound.[17] Ultrasound-based therapies have several distinct advantages, which includes being non-invasive, portable, and targeted. Ultrasound can induce oscillation, rupture, and collapse of the double emulsion droplets, which releases the payload from the core of the emulsion.[17-19] The perfluorohexane shell undergoes a phase change during vaporization causing a change from liquid to gas.[17] It is theorized, due to anisotropic pressures, that inertial cavitation will occur, which can cause transient pores in nearby cell membranes and enable transport of therapeutic compounds directly into the cytoplasm of cells (fig. 2). [20-23]

AS1411 was initially discovered by Dr. Paula Bates and colleagues at the University of Alabama-Birmingham. Since being discovered, AS1411 has completed clinical trials for treatment of acute myeloid leukemia and metastatic renal cell carcinoma.[24, 25] For this project, AS1411 aptamer, a 26-mer oligonucleotide, is bound 
to the surface of the double emulsion droplet in order to target nucleolin. In normal cells, nucleolin is a multifunctional protein located predominantly in the nucleolus; however, it can also be found in the cytoplasm and as a surface receptor.[26-30] Deregulation occurs in tumor cells causing an over-expression of nucleolin surface receptors in many tumor cell lines.[27, 29] AS1411 binds to nucleolin with high affinity and is taken into the cell through mediator receptor macropinocytosis.[26, 30]

Prior studies have shown that ultrasound can trigger release of therapeutics from double emulsions and AS1411 can specifically target cancer cells. Conjugating AS1411 to double emulsions will allow effective targeting of cancer cells that overexpress nucleolin surface receptors. Once bound, ultrasound can be applied to induce transient pores, allowing molecular therapeutics to enter the cell. Therefore, the objective of this project was to combine these findings and assess the effect of using ultrasound to induce drug release from AS1411-targeted double emulsions in cancer cells. 


\section{Targeted Drug Delivery to Cancer Cells in Static Culture Conditions}

\section{A. Motivation}

When investigating ultrasound-responsive double emulsions, prior research studies have not explored the potential ability to chelate an aptamer to ultrasound-responsive double emulsion droplets. This led our team to want to investigate this area further. Aptamers have the ability to actively target certain cell types based off their phenotype.[4] In our case, we selected an aptamer that has the ability to target nucleolin-expressing cancer cells, such as the human triple-negative breast cancer (TNBC) cell line MDA-MB-231, which we selected for our experimental testing. Our objective was to evaluate whether chelating a tumor-targeting aptamer onto the double emulsion improved uptake of the payload in the cells compared to untargeted particles. Secondly, we wanted to see if ultrasound improved the delivery of the payload compared to control samples which did not receive application of ultrasound.

\section{B. Methods}

i.) Generation of Polydisperse Targeted Double Emulsions

We placed $375 \mu \mathrm{L}$ of deprotected AS1411 maleimide solution in a microcentrifuge tube. By deprotecting AS1411, it will allow the thiol group to react with the maleimide group on polar head of the phospholipid surfactant. The thiol group on AS1411 was deprotected with 10mM of (tris)(2-carboxyethyl)phosphine) (TCEP) for 1 hour and immediately added to lipid solutions for overnight incubation at $4{ }^{\circ} \mathrm{C}$ to allow the reaction 
to occur. For untargeted control samples we placed $300 \mu \mathrm{L}$ of $2 \%$ maleimide solution and $75 \mu \mathrm{L}$ of PBS in a microcentrifuge. Both solutions were used as the surfactant. AS1411 has been shown to target nucleolin receptors while the maleimide solution without AS1411 has no functional tumor-targeting moieties. We agitated the solutions using a micropipette to ensure thorough mixing. We then added $375 \mu \mathrm{L}$ of Poloxamer 188 into both solutions and stored them at $4{ }^{\circ} \mathrm{C}$. We placed $400 \mu \mathrm{L}$ of $100 \mathrm{mg} / \mathrm{mL}$ fluorescein sodium salt in deionized water and $800 \mu \mathrm{L}$ of perfluorohexane $(\mathrm{PFH})$ in a $15 \mathrm{~mL}$ centrifuge tube. Fluorescein sodium salt was selected over chemotherapeutic drugs since it has a higher fluorescence level, allowing for better quantitative analysis. Using a $20 \mathrm{kHz}$ sonicator, we set the amplitude to $30 \%$ for 30 seconds and sonicated the samples while on an ice bath. We removed the surfactants from $4{ }^{\circ} \mathrm{C}$ storage and placed the entire contents of each solution in separate $2 \mathrm{~mL}$ glass vials. We pipetted the fluorescein sodium salt/PFH solution up and down multiple times to ensure that the solution was well mixed. We then pipetted $250 \mu \mathrm{L}$ into each surfactant solution. A septum cap was crimped onto each glass vial, and the solution was amalgamated for 45 seconds. We removed each vial from the amalgamator and left it to sit for 20 minutes to allow the double emulsions to settle at the bottom of the vial.

\section{ii.) Treatment of TNBC Cells at Physiological Conditions}

Seven different treatments were used on MDA-MB-231 cells. The objective of this study was to observe if a dose dependent effect occurred, to determine the effect of a targeting aptamer on uptake of molecular compound, and to determine the effect of ultrasound on release and delivery of the molecular compound from double emulsions. 
Three different doses were used with AS1411-conjugated double emulsions and untargeted double emulsions: low dose $(25 \mu \mathrm{L})$, medium dose $(50 \mu \mathrm{L})$, and high dose $(100 \mu \mathrm{L})$. Each dose was added at a 1:20 ratio with PBS before treatment to ensure double emulsions were well distributed amongst cells. Treatments were added to a petri dish containing MDAMB-231 cells. Following treatment with compound, the petri dishes were placed back in the $\mathrm{CO}_{2}$ incubator for 4 hours at $37{ }^{\circ} \mathrm{C}$. Following 4 hours in the incubator, groups were treated with ultrasound or no ultrasound based on experimental group specification.

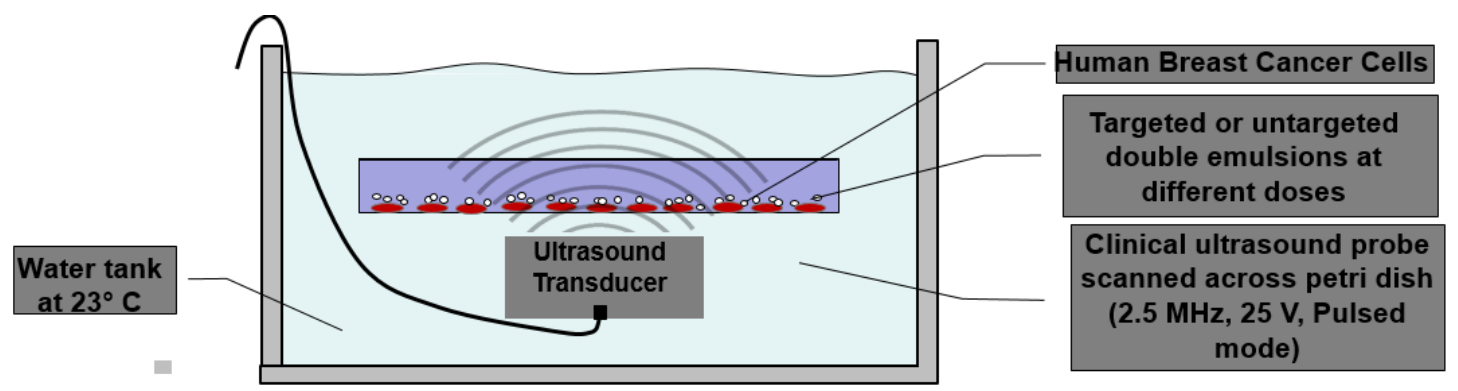

Figure 3- schematic of experimental setup for treatment of MDA-MB-231 using ultrasound to vaporize double emulsions.

In groups that were treated with ultrasound, the petri dish was scanned across the ultrasound beam to ensure triggered release of molecular compound occurred, shown in Figure 3. Ultrasound pulses were delivered with an ultrasound imaging system $(2.5 \mathrm{MHz}$, ATL P4-1 probe, Verasonics Vantage 64LE system, $3 \mathrm{MPa}$ peak negative pressure). Following ultrasound treatment, cells were washed with PBS. Cells were trypsinized to remove cells that were adhered to the bottom of the petri dish. Media was used to neutralize the trypsin, and the cells were placed in a vial. Cells were spun down at $1000 \mathrm{rpm}$ for 5 minutes using a centrifuge. Supernatant was removed and cells were resuspended in $1 \mathrm{~mL}$ before assessing fluorescein uptake using flow cytometry. All data was normalized to AS1411 conjugated low dose treatment. Statistical analysis was performed in Minitab 
using a two-way ANOVA. For samples imaged with scanning electron microscopy (SEM), cells were immediately fixed ( $<1 \mathrm{~min})$ in $2.5 \%$ glutaraldehyde after ultrasound treatment. iii.) Treatment of TNBC with Ultrasound

The objective of this study was to determine the effect of ultrasound on release and delivery of the molecular compound from double emulsions. Two groups were used for the treatment of MDA-MB-231 cells: double emulsions $(70 \mu \mathrm{L})$ vaporized with ultrasound and double emulsions $(70 \mu \mathrm{L})$ with no treatment. Once MDA-MB-231 cells were treated with double emulsions, the petrie dishes were stored at $21^{\circ} \mathrm{C}$ for 5 minutes. This allowed all the double emulsions to sink to the bottom of the petrie dish, and it was stored at $21^{\circ} \mathrm{C}$ to reduce the amount of passive release of the payload. In the group that was treated with ultrasound, the petri dish was scanned across the ultrasound beam to ensure triggered release of molecular compound occurred, shown in Figure 3. Ultrasound pulses were delivered with an ultrasound imaging system (2.5 MHz, ATL P4-1 probe, Verasonics Vantage 64LE system, 3 MPa peak negative pressure). Following ultrasound treatment, cells were washed with PBS. Cells were trypsinized to remove cells that were adhered to the bottom of the petri dish. Media was used to neutralize the trypsin, and the cells were placed in a vial. Cells were spun down at $1000 \mathrm{rpm}$ for 5 minutes using a centrifuge. Supernatant was removed and cells were resuspended in $250 \mu \mathrm{L}$ before assessing fluorescein uptake using flow cytometry. All data was normalized to double emulsions where ultrasound was not applied. Statistical analysis was performed in Minitab using student T-test.

C. Results and Discussion 
Tumor-targeted double emulsions versus non-targeted double emulsions showed no statistically significant difference $(\mathrm{p}=0.628)$. Previous studies have shown that AS1411 has the ability to target and induce uptake through micropinocytosis in cancer cells. Multiple factors could explain the reasoning for the lack of a statistical difference. The most plausible explanation is that double emulsions sink in cell culture due to their higher density compared to the surrounding media, which allows the emulsion droplets to contact the cells without being dependent on ligandreceptor binding. For future studies, a dynamic flow model could be used to prevent

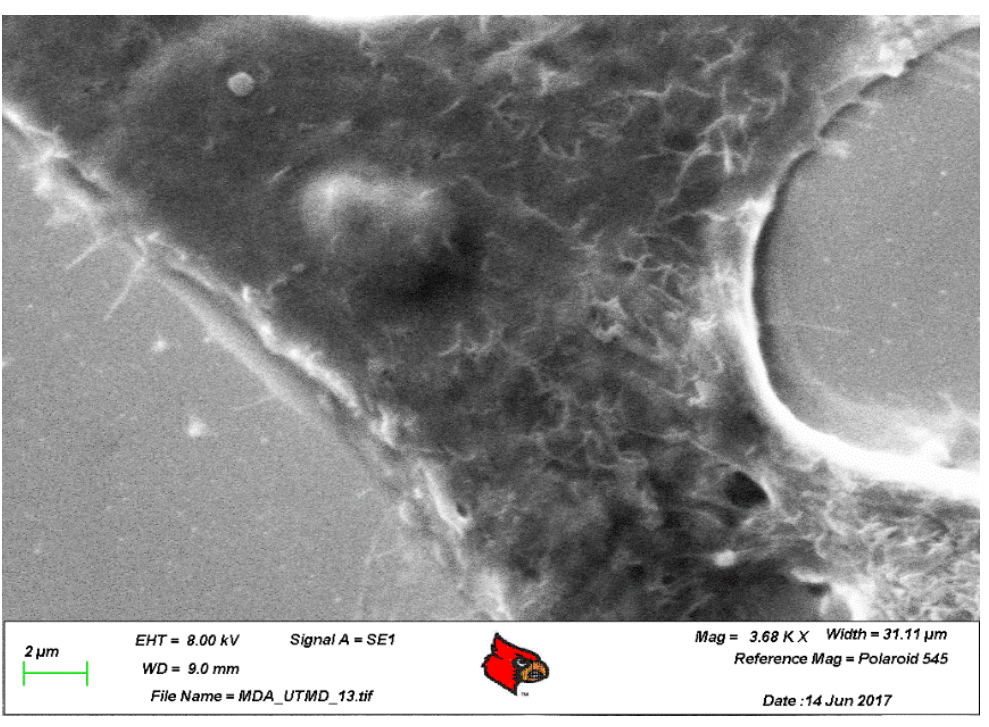

emulsions from accumulating at the bottom of the container before treating with ultrasound. 
Ultrasound treatment also did not show any statistically significant increase compared to untreated control groups $(\mathrm{p}=.054)$. Additional testing is necessary to determine if ultrasound has an effect on uptake. In fact, in this study it appears that ultrasound may actually have a detrimental effect on uptake. This may be an artifact of the experimental setup in which high passive uptake of molecular compounds occurs. In this case, ultrasound treatment may be inducing leakage of the molecular compounds from the cells. The purpose of ultrasound treatment was to cause a phase change in perfluorocarbon allowing the release of the compound from the double emulsion droplets. It was theorized that double emulsions proximal to a cell

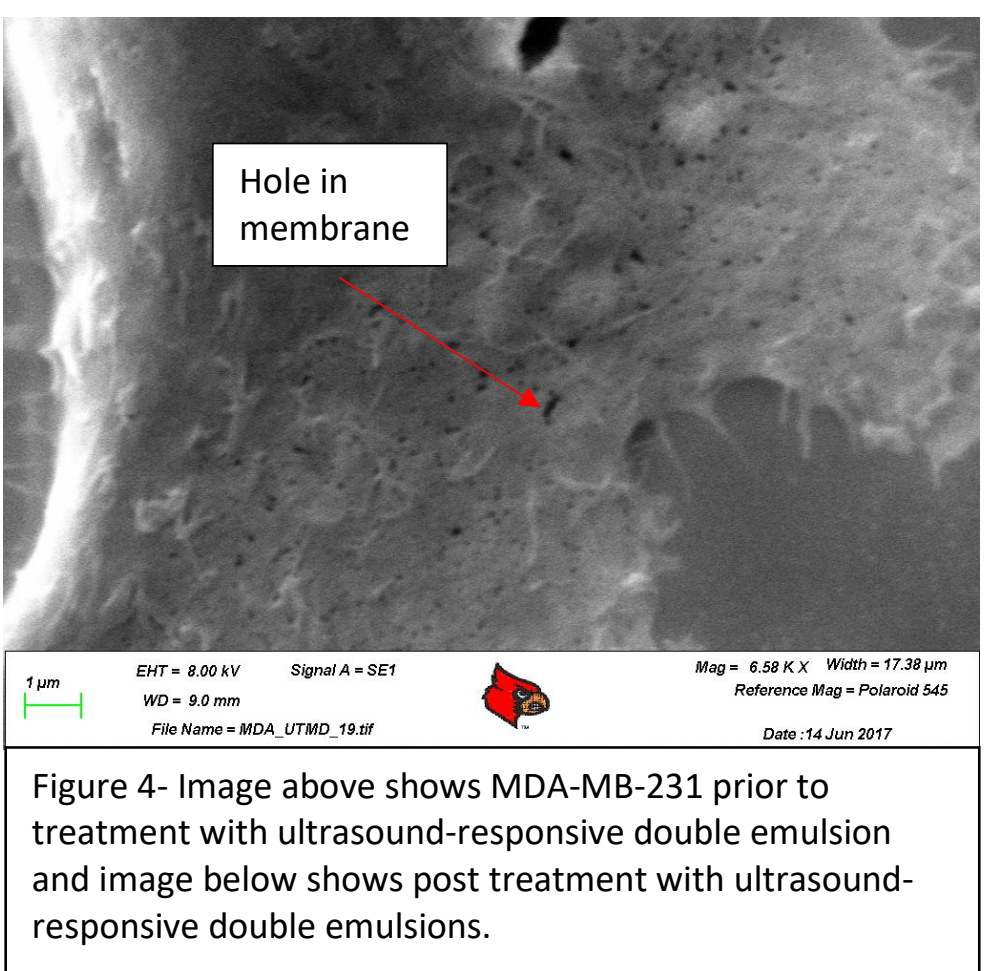
will induce a collapse of the perfluorocarbon phase which can potentially facilitate rapid transport of payload directly to the cell by causing transient pores in the cell's membrane. This led us to further investigate the induced interaction between the double emulsion and cell membrane that may be caused by ultrasound treatment. Our finding, shown in figure 4 , indicates the presence of transient pores in the cell membrane following treatment of ultrasound. This confirms that ultrasound could have a potential use as a therapeutic delivery method from double emulsions. In this circumstance, however, double emulsions 
had the ability to interact with the cancer cells for approximately four hours. This is ample time for endocytosis-mediated uptake by the cell. Thus, a high concentration of payload is already inside the cell. When vaporization occurs, the payload can actually leave the cell by the newly formed transient pores.

High dose treatment was shown to be statistically different compared to low dose $(\mathrm{p}<.01)$ and medium dose $(\mathrm{p}<.01)$. No statistical difference was found between low dose and medium dose $(\mathrm{p}=0.074)$, although there was a trend toward significance and a larger 
sample size may have revealed a statistical difference (fig. 5).

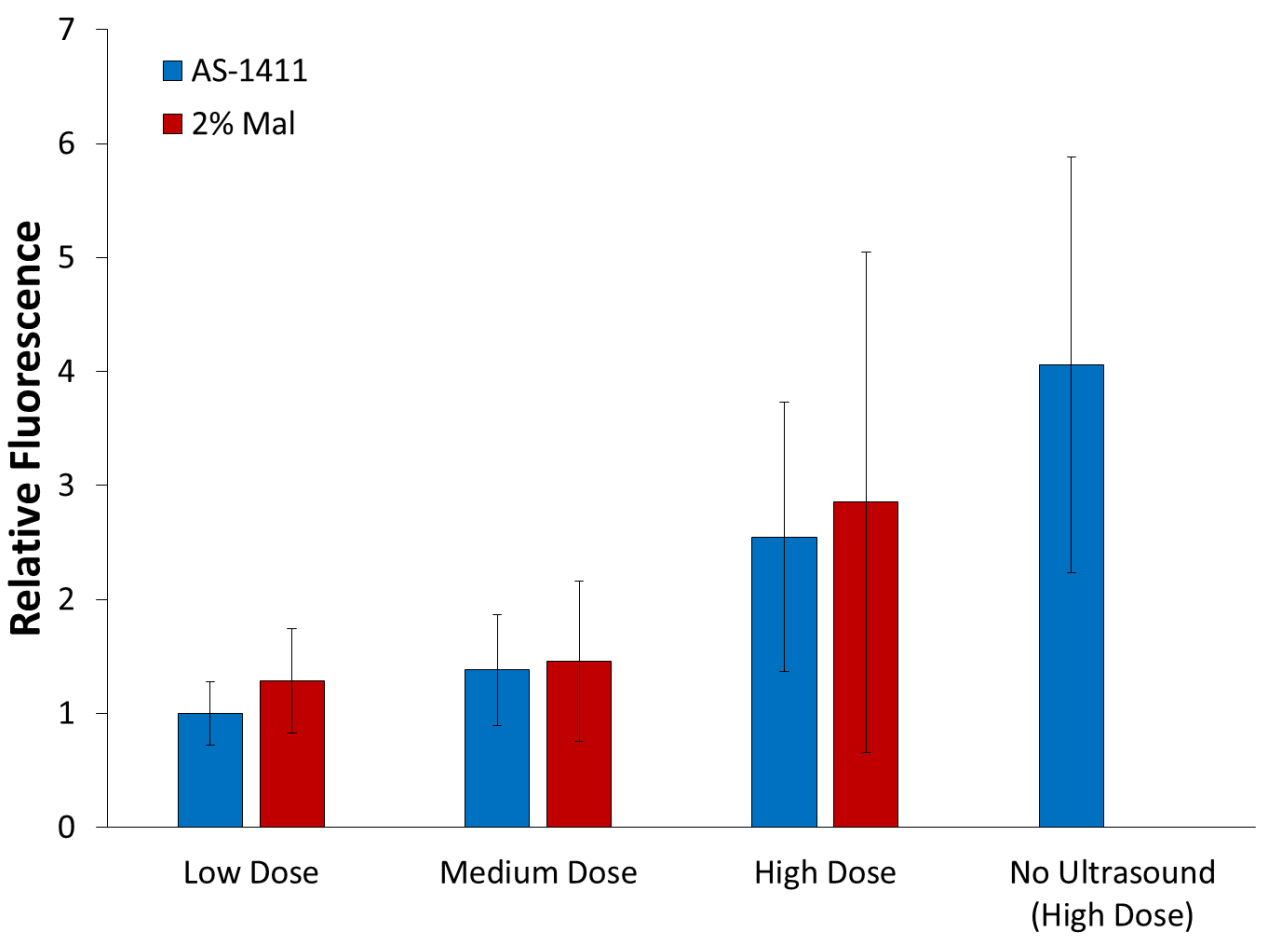

Figure 5- Shows the relative fluorescence normalized to AS1411 low dose treatment. Low, medium, and high dose were all treated with ultrasound, excluding the no ultrasound (high dose) group.

The results of this study indicated that the double emulsions were not stable after 4 hours of incubation at $37^{\circ} \mathrm{C}$ and spontaneously released their payload prior to ultrasound treatment. In addition, molecular compound (i.e. fluorescein) was taken up by cells even without AS1411 targeting, indicating non-specific delivery (likely by endocytosis) in the static culture system. The SEM image showing pores in the membrane is a positive finding since this indicates that molecular compounds could enter the cytosol of the cancer cell more easily. Nevertheless, due to limitations of the static cell culture system and double emulsion stability we did not observe any significant differences in uptake of the molecular compound by cells between experimental groups. 
Following the results, surfactants were investigated to determine the ability for it to stabilize the particle to reduce the passive release of the payload, which is discussed in further detail in the next section. A study was performed to further analyze the effect that ultrasound had on uptake of the payload. Due to the density of the double emulsion, we observed that double emulsions sank to the bottom of the container within the first minute (fig. 6). A group was treated with ultrasound after 5 minutes and was compared to a group that was not treated with ultrasound to determine

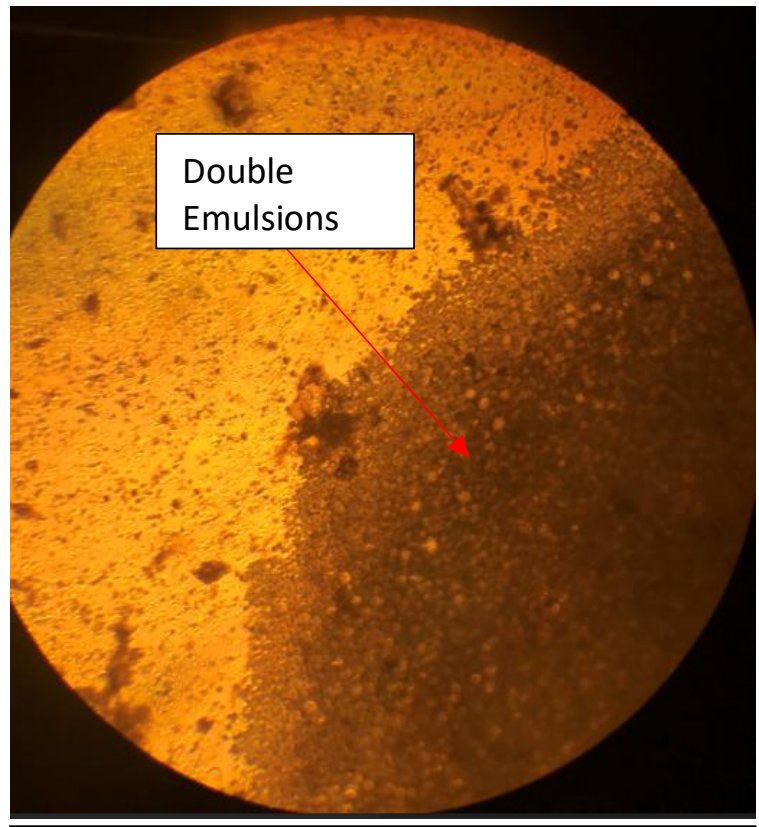

Figure 6- Image taken 1 minute after placing double emulsions in culture.

if ultrasound had an impact on uptake of molecular compound. It was determined that ultrasound treatment induced more uptake of fluorescein $(\mathrm{p}<0.05)$ (fig. 7).

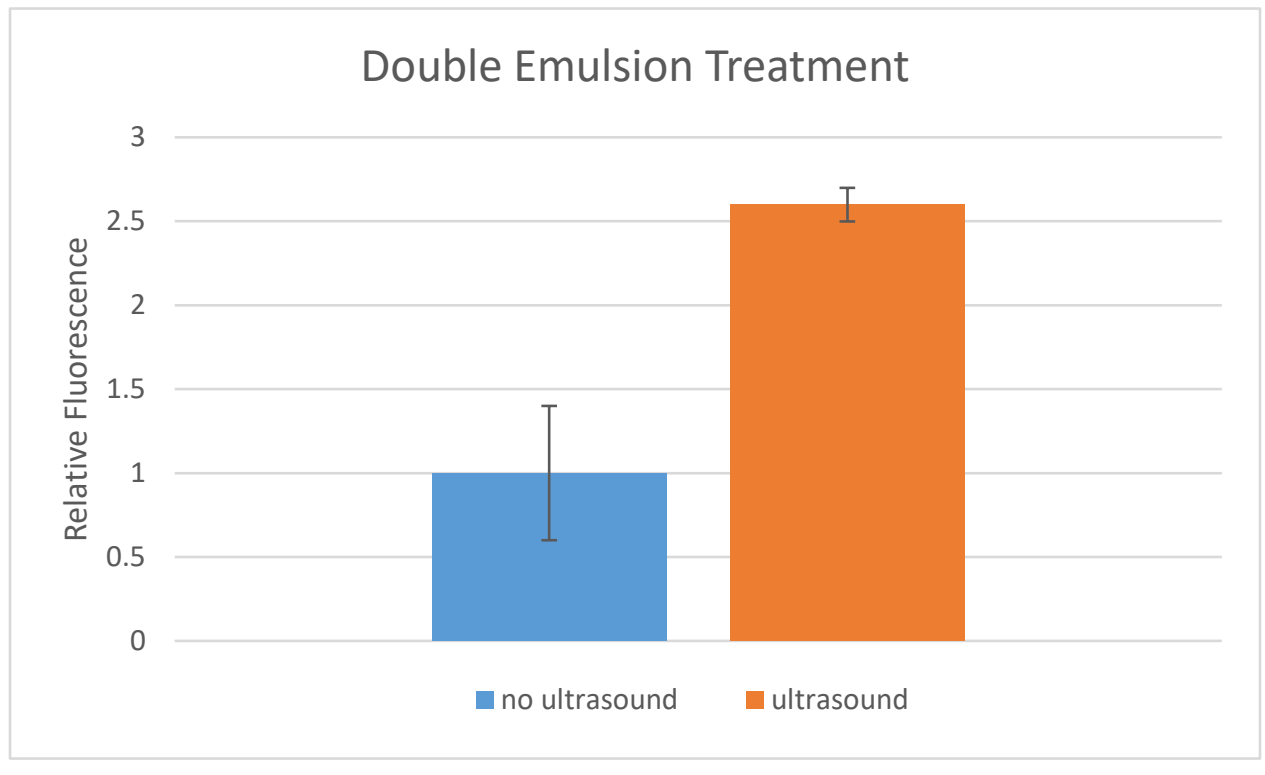

Figure 7- compares the effect of ultrasound on fluorescein uptake. All data was normalized to no ultrasound treatment. 
III. Characterization of Surfactant Effects on Double Emulsion Stability

\section{A. Motivation}

Based off of the synthesis techniques described above, double emulsions could not be stored long term for usage. Double emulsions had to be synthesized and used for experiments right away. In the next experimental study, different surfactant types, concentrations, and temperatures were tested to see the effect on the release profile of the double emulsions and increase the storage shelf-life of double emulsions. For double emulsions to be translated to the clinic, they must be able to be synthesized and stored for a duration of time prior to treatment without releasing the payload. With a more stable surfactant, a slower release profile will take place. Also, the effect of temperature on double emulsion stability was investigated to determine leakage of the internal payload over time at different temperatures.

\section{B. Methods}

i.) Synthesis of Double Emulsions

We evaluated three different surfactants for experimental testing: Poloxamer 188 (Sigma-Aldrich), FluorN561 (Cytonix), and FluorN562 (Cytonix). Poloxamer 188 is a nonionic copolymer composed of a hydrophobic chain flanked by two hydrophilic chains. Poloxamer 188 has been commonly used as a surfactant for research due to its biocompatibility. However, for double emulsion use, it was unclear if it would provide 
enough stability for drug delivery applications. Thus, other surfactants were investigated. FluorN561 and FluorN562 are non-ionic, ethylene glycol-based fluorosurfactants and have the same structural backbone (fig. 8). Fluor561, commonly denoted as N561, contains one perfluro group and three polyethylene glycol groups, and FluorN562, commonly denoted as N562, contains two perfluoro groups and two polyethylene glycol groups. Both of these surfactants appeared advantageous since both are expected to be biocompatible and had longer chains compared to Poloxamer 188. The longer chain lengths may reduce coalescence of the drug delivery vehicles.

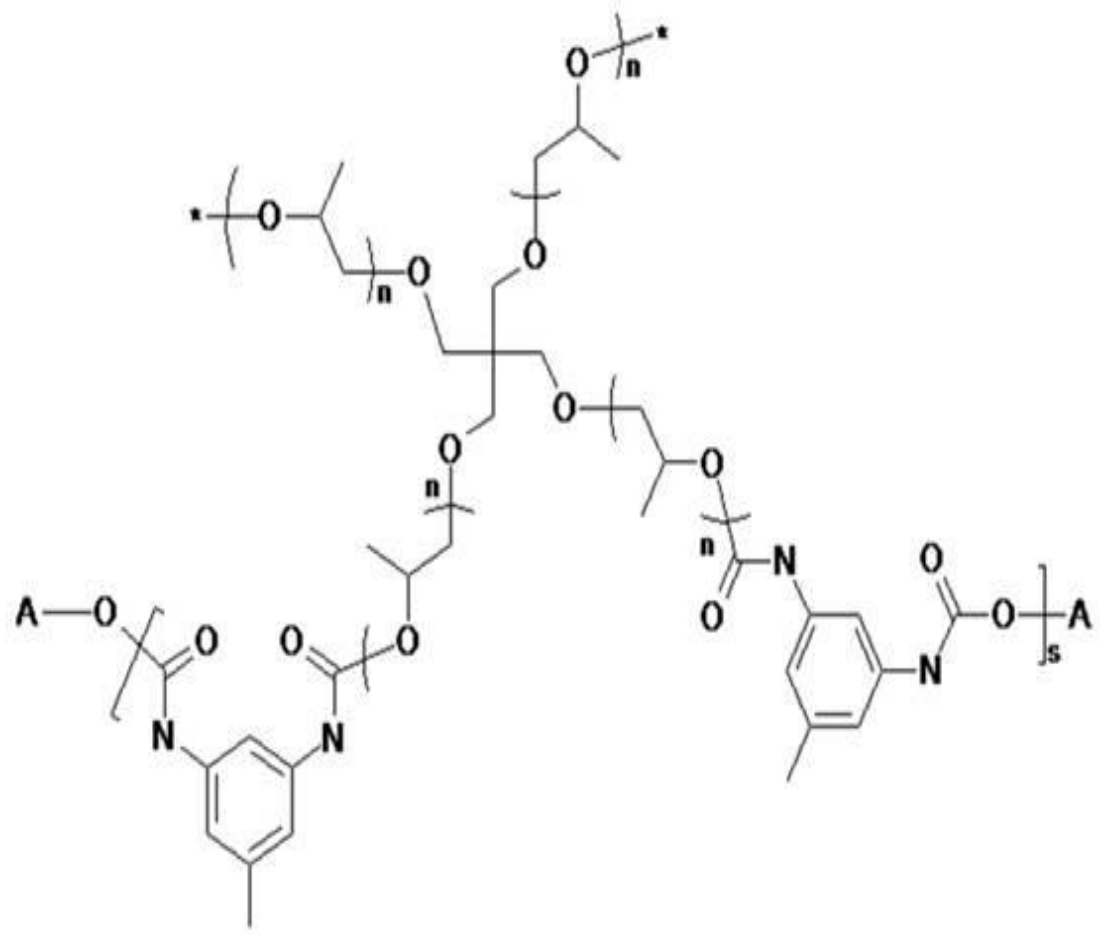

Figure 8- shows the structural backbone of FluorN561 and FluorN562. Functional groups, which are either hydrophobic or hydrophilic in nature, are attached to the $A$ and * terminals. 
We diluted each solution to three different concentrations for testing. Poloxamer 188 concentrations were tested at 1\%,2.5\%, and 5\% (w/v). N561 and N562 concentrations were tested at $0.2 \%, 0.5 \%$, and $1 \%(\mathrm{w} / \mathrm{v})$. Stock solutions were prepared at $10 \%(\mathrm{w} / \mathrm{v})$ for Poloxamer 188 and 2\% (w/v) for FluorN561 and FluorN562. For the lowest concentration of each solution, $200 \mu \mathrm{L}$ was added to $1.8 \mathrm{~mL}$ of PBS. For the middle concentration, 500 $\mu \mathrm{L}$ was added to $1.5 \mathrm{~mL}$ of PBS. For the highest concentration, $1 \mathrm{~mL}$ was added to $1 \mathrm{~mL}$ of PBS. In a $2 \mathrm{~mL}$ glass vial, $200 \mu \mathrm{L}$ of fluorescein deionized water solution at a concentration of $200 \mathrm{mg} / \mathrm{mL}$ was added to $1.5 \mathrm{~mL}$ of diluted surfactant. A septum cap was crimped onto the glass vial and the solution was amalgamated for 45 seconds. Following amalgamation the solution was left undisturbed on the bench for 20 minutes at room temperature. This allowed the double emulsions to settle on the bottom of the solution.

\section{ii.) Washing Procedure for Double Emulsions}

Double emulsion washing steps were included to remove unencapsulated compounds from the solution. Prior to removing the supernatant that contained the free compounds, additional solutions were made for washing the emulsions. Resuspension in PBS is a common methodology for most methods; however, it was discovered that in a PBS solution without surfactants, the double emulsion would release its compound very quickly. To prevent that from occurring, we prepared $5 \mathrm{~mL}$ solutions of $1 \%, 2.5 \%$, and $5 \%$ Poloxamer $188,5 \mathrm{~mL}$ solutions of $0.2 \%, 0.5 \%$, and $1 \%$ FluorN561, and $5 \mathrm{~mL}$ solutions of $0.2 \%, 0.5 \%$, and $1 \%$ FluorN562 using PBS. We removed $1 \mathrm{~mL}$ aliquots of each surfactant concentration and pipetted it into a separate vial. We then removed double emulsions of each solution and gently pipetted them into corresponding vials that contained $1 \mathrm{~mL}$ of 
surfactant. We allowed 5 minutes for the double emulsions to settle, then we remove the supernatant from the solution. We carefully washed an additional three times and removed the supernatant, allowing double emulsions to settle for 3 minutes each time.

\section{iii.) Release Profile of Double Emulsion}

For each surfactant type and concentration, three samples were prepared. Each sample was covered with foil to prevent photobleaching of the fluorescence in the solution. For N562 and Poloxamer 188 samples, the three concentrations were stored at three different temperatures to observe the effect that temperature has on the release profile for each emulsion type. N561 was not sampled since stable formation of double emulsions did not occur with this surfactant. The conditions were $4{ }^{\circ} \mathrm{C}, 21{ }^{\circ} \mathrm{C}$, and $37{ }^{\circ} \mathrm{C} .20 \mu \mathrm{L}$ aliquots were taken from the supernatant of each sample and placed in a 96 well plate. We then added $180 \mu \mathrm{L}$ of deionized water to dilute each sample in the 96 well plate. Fluorescence was measured with a spectrofluorometer using an excitation wavelength of $488 \mathrm{~nm}$ and an emission wavelength of $520 \mathrm{~nm}$. The measurements were acquired at $1 \mathrm{hr}, 2 \mathrm{hr}, 4 \mathrm{hr}, 24$ $\mathrm{hr}, 72 \mathrm{hr}$, and $72 \mathrm{hr}$ post treatment. All data was normalized to the last data point taken. 
C. Results and Discussion

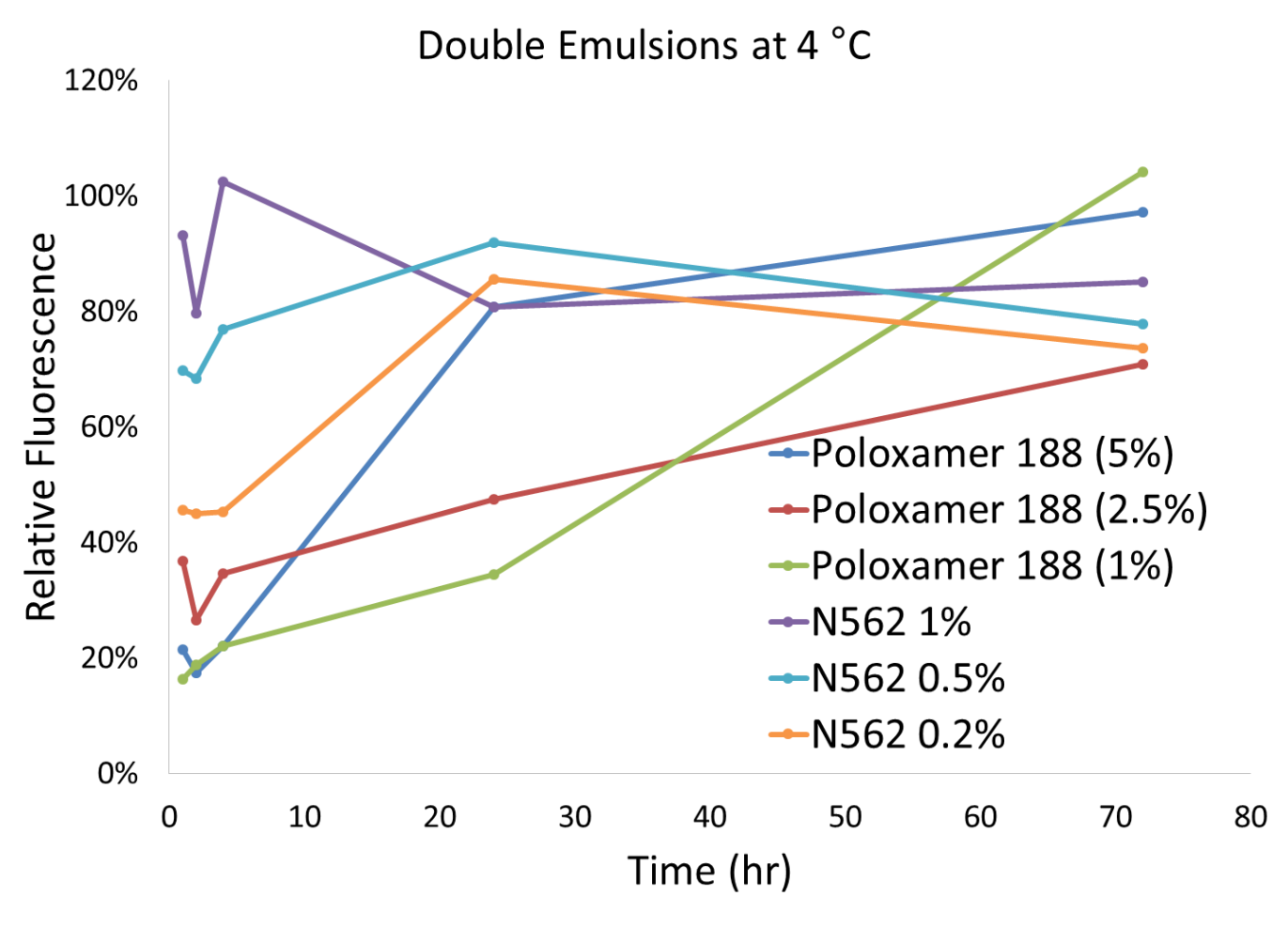

Figure 9- shows the release profile for Poloxamer 188 and N562 at different surfactant concentrations up and being stored at $4^{\circ} \mathrm{C}$. All data was normalized to 72 hour post-treatment with ultrasound. 


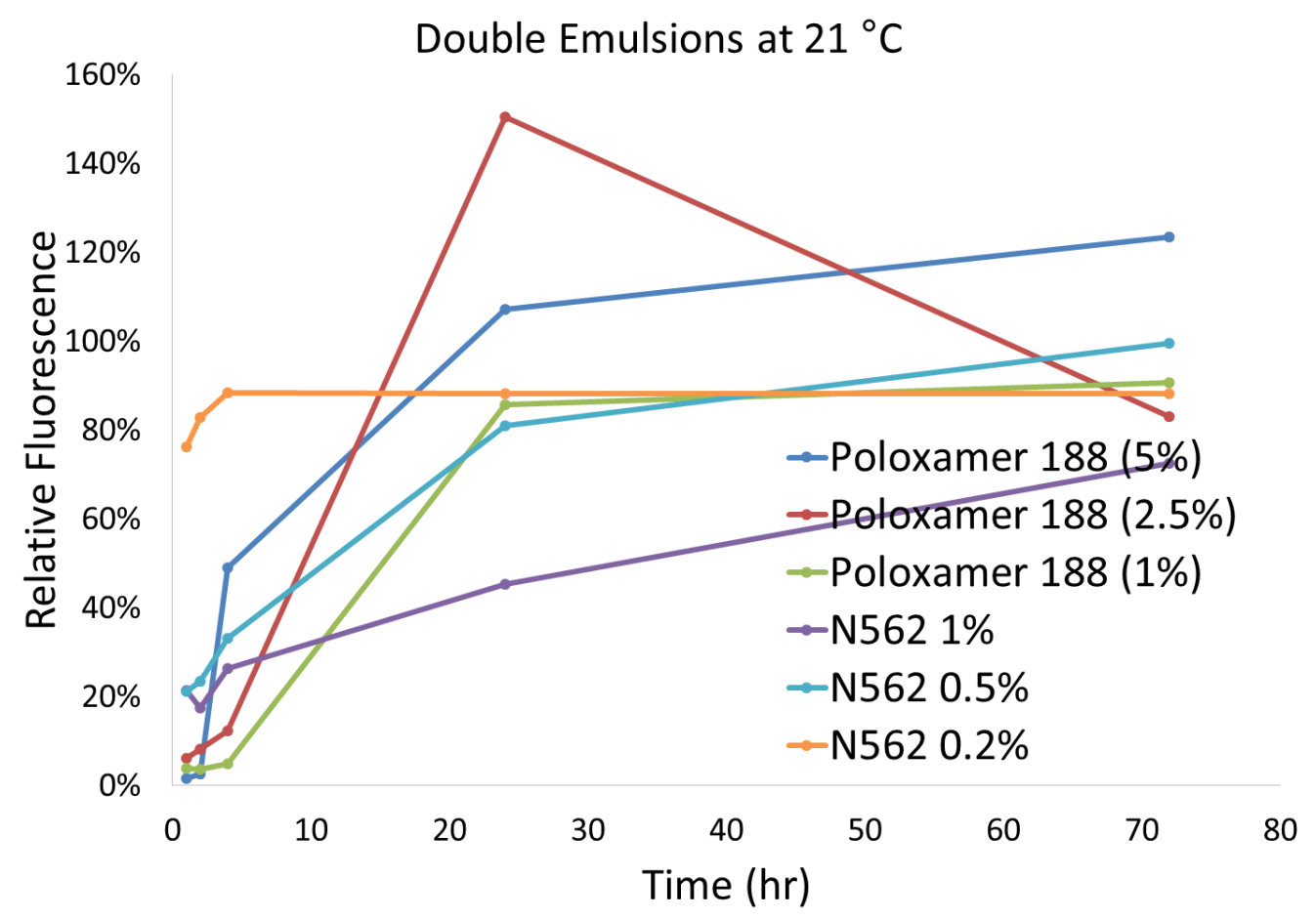

Figure 10-shows the release profile for Poloxamer 188 and N562 at different surfactant concentrations up and being stored at $21^{\circ} \mathrm{C}$. All data was normalized to 72 hour post-treatment with ultrasound. 


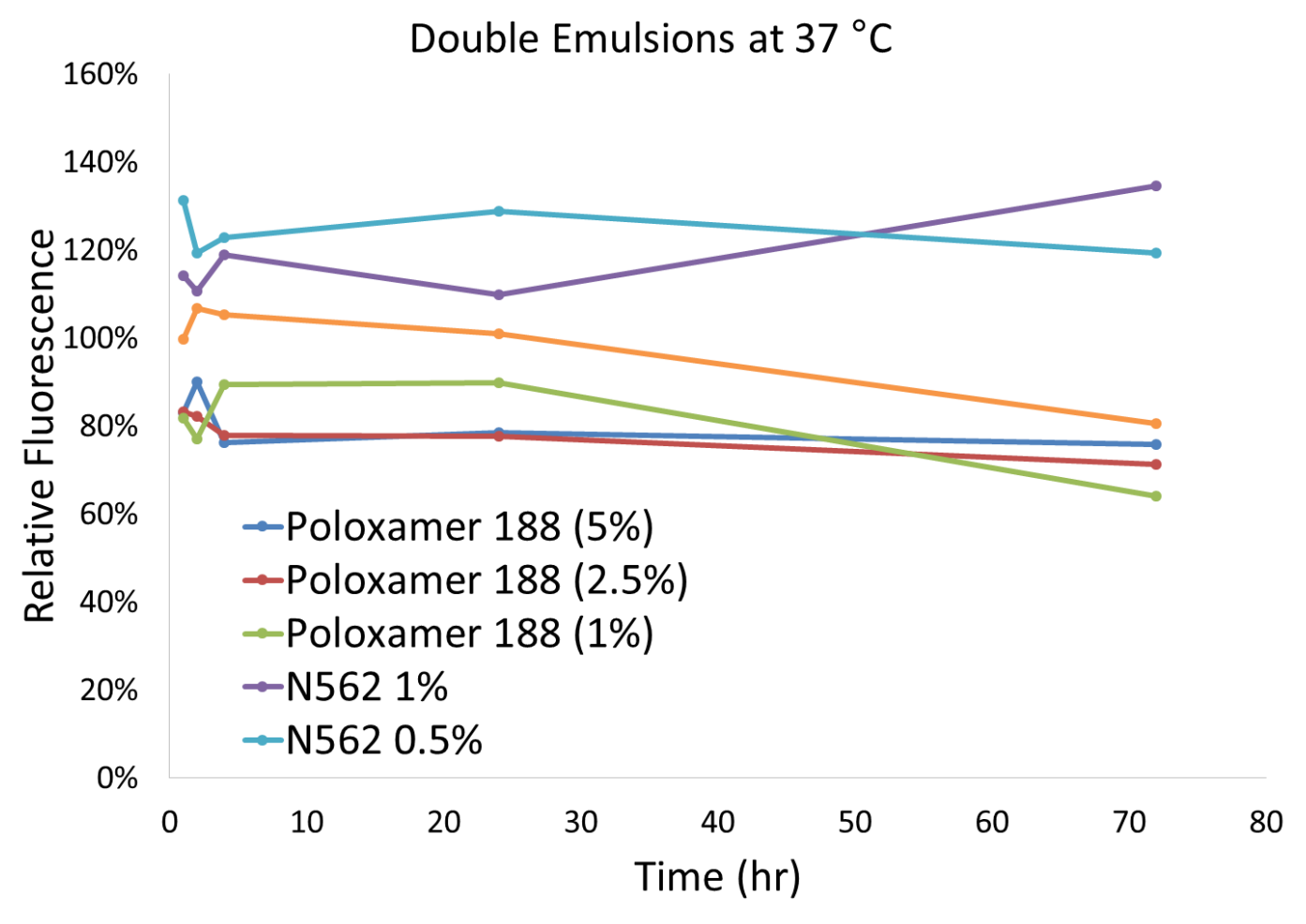

Figure 11- shows the release profile for Poloxamer 188 and N562 at different surfactant concentrations up and being stored at $37^{\circ} \mathrm{C}$. All data was normalized to 72 hour post-treatment with ultrasound.

Initial testing showed that temperature may have a significant effect on the passive release profile of the emulsion droplets but was not statistically significant. Passive release profile occurs when the payload crosses the perfluorohexane shell into solution prior to the vaporization of the double emulsions. For Poloxamer 188 and N562 emulsions at $37{ }^{\circ} \mathrm{C}$ (fig. 11), almost all of the fluorescein was released from the double emulsions within 1 hour. This suggests that an additional surfactant is necessary to have a more sustained release profile at that temperature. For Poloxamer 188 and FluorN562 emulsions at $4{ }^{\circ} \mathrm{C}$ and $21^{\circ} \mathrm{C}$ (fig. $9 \& 10$ ), a slower release profile occurred. This suggests that either $4{ }^{\circ} \mathrm{C}$ or $21{ }^{\circ} \mathrm{C}$ may be used for emulsion storage before treatment. FluorN561 is not a viable option 
as a surfactant for double emulsions due to the inability for stable double emulsions to be formed.

Additional testing was performed to further examine the effect that surfactant and temperature has on release profile of double emulsions. FluorN562 performed better than Poloxamer188 at each temperature $(\mathrm{p}<0.05)$. FluorN562 showed a slower release profile (fig. 12). In order for ultrasound-responsive, targeted double emulsions to be used

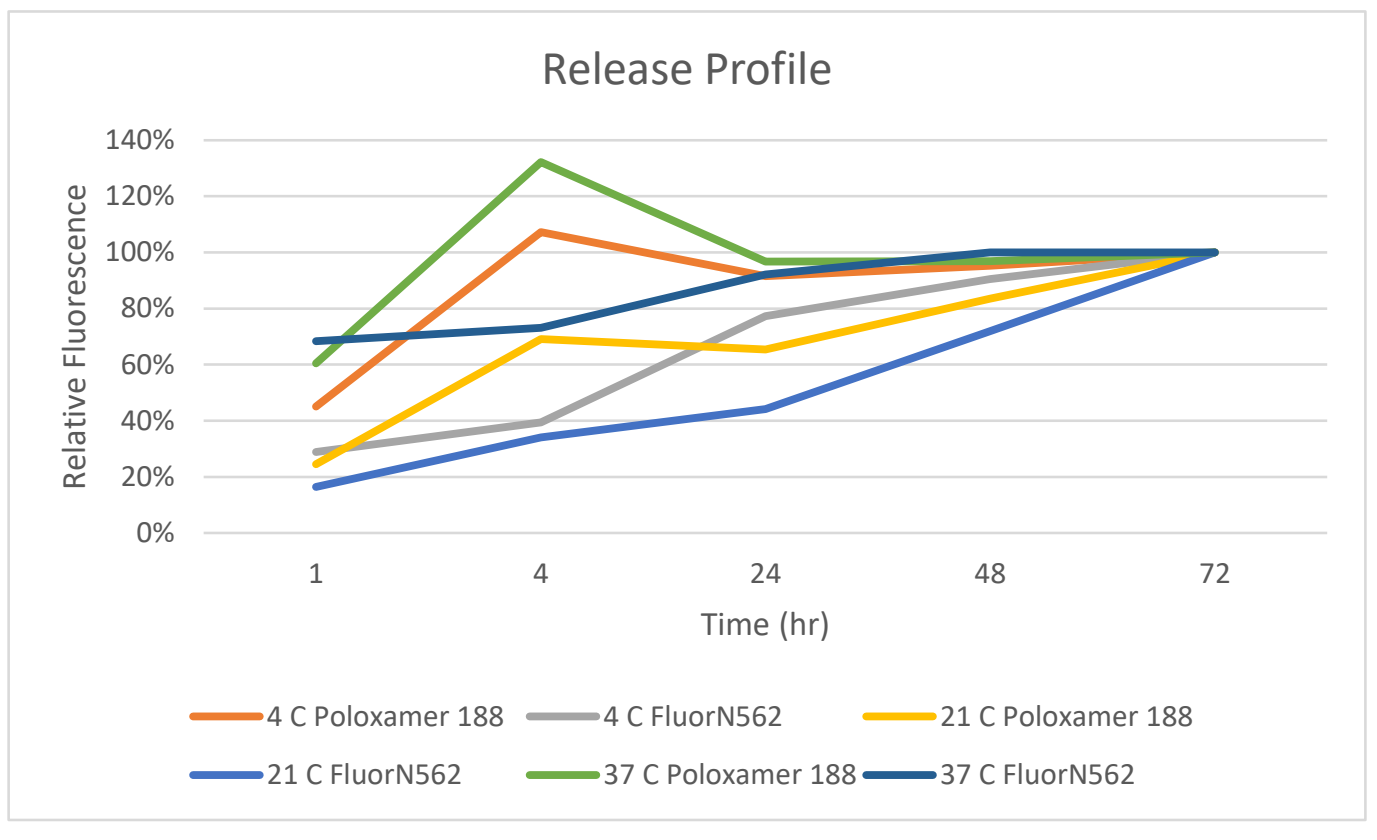

Figure 12- Shows the relative fluorescence for each surfactant and corresponding temperature. Each data set was normalized to their last measured point at 72 hours.

clinically, a slow release profile is needed so that the emulsion remains intact until it is vaporized by ultrasound at the target site. This will allow a potentially high amount of payload to be delivered to the tumor. FluorN562 at $21^{\circ} \mathrm{C}$ exhibited the best release profile ( $\mathrm{p}<0.05$ ). Although $21^{\circ} \mathrm{C}$ appeared to be optimal, it is important to consider that cooling and heating of perfluorohexane from $21^{\circ} \mathrm{C}$ may have had an impactful role on the release profile. It was observed as time progressed, the surfactant and solution and would separate, potentially altering the release profile of double emulsions. 
To improve double emulsion stability, multiple parameters should be considered. Double emulsions tend to coalesce without a surfactant. To prevent coalescing from occurring, surfactants are used. Surfactant type and chain length are both important properties to consider. When selecting a surfactant, non-ionic surfactant appear to be suitable since it contains no charge and contains polar head groups, which prevents interaction between groups. Chain length is also critical. By increasing chain length, interaction between the perfluorohexane shell should be reduced, reducing the amount of double emulsions that coalesce.

IV. Microfluidic Synthesis of Monodisperse Double Emulsions

\section{A. Motivation}

Perfluorocarbon-based double emulsions could have a profound effect clinically for targeted delivery of therapeutic compounds. However, prior limitations have included inadequate droplet size and heterogeneity of double emulsion suspensions. In order for double emulsions to be used clinically, the emulsion droplets must be smaller than 5 microns to travel through the capillaries. Microfluidic devices were developed to synthesize monodispersed double emulsions that are under 5 microns. Monodisperse double emulsions have many advantages compared with polydisperse double emulsions. 
With monodisperse double emulsions, the size of each droplet in the emulsions is known and can be reproduced consistently, thus it is possible to perform quality control to determine if the emulsions could be used clinically based on the size, but with polydisperse double emulsions there can be a large variance in size and the distribution can vary between batches.

\section{B. Design}

Multiple components were considered in the design of the microfluidic system, which included flow type and channel size. Flow characteristics have an important impact on output of double emulsions. If flow is turbulent, steady production of double emulsions will not occur since coalescence of droplets is more likely to occur. Channel size is an important factor in double emulsion production. If channels are too small, clogging is likely to occur. We modified a special design recently described in the literature (fig. 13),[31] which employs a double-layer format to enable formation of very small double emulsions (less than 10 microns in diameter) through an orifice.

Figure 13- Schematic of microfluidic device used to create monodispersed double emulsions.

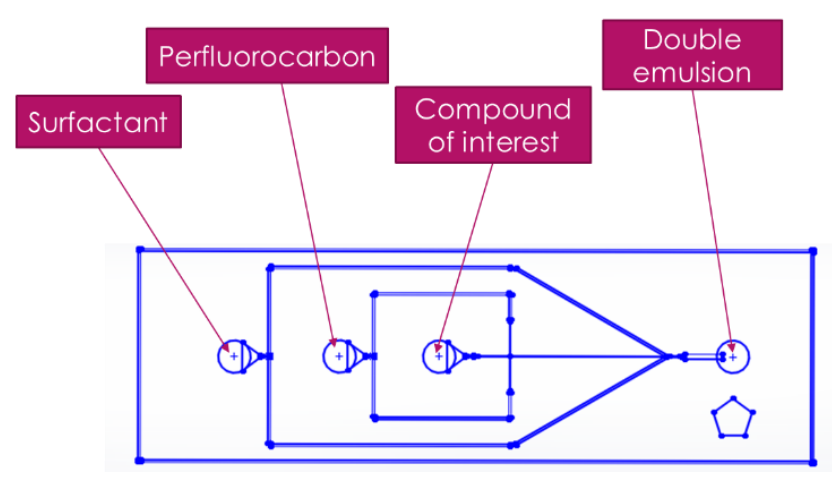

C. Methods 


\section{i.) Fabrication of Microfluidic Devices}

The SU8 master was fabricated on a silicon wafer at the UofL Micro/Nano Technology Center using standard photolithography techniques. PDMS-based microfluidic devices were fabricated using previously established methods. Briefly, $60 \mathrm{~g}$ of silicone base and $6 \mathrm{~g}$ of curing agent was added to a cup and was mixed thoroughly using a stir stick. The cup containing the mixture was degassed by placing it in the desiccator until air bubbles were no longer visible. All of the PDMS was poured over the SU8 master and placed back into the desiccator to remove any remaining air bubbles. Following the removal of all bubbles, the device was baked for 2 hours at $60{ }^{\circ} \mathrm{C}$ in a lab oven. The PDMS was carefully peeled off the SU8 master before removing PDMS devices. Each individual device was cut from the PDMS block using a razor blade. The microfluidic inlets and outlets were punched in the PDMS using a $2 \mathrm{~mm}$ biopsy puncher. Once devices were prepared, they were treated with oxygen plasma $\left(100 \mathrm{~V}\right.$ for $60 \mathrm{~s}, 0.5$ atm of $\left.\mathrm{O}_{2}\right)$ and immediately bonded onto glass microscope slides.

\section{ii.) System Preparation}

The microfluidic device was placed on the stage of an inverted microscope coupled with a digital camera that can take frames less than $100 \mu$ s apart. Syringes were mounted on a syringe pump for continuous infusion into the microfluidic device. We found that syringes of $10 \mathrm{~mL}$ or larger were optimal. Syringe were primed before attaching approximately $45 \mathrm{~cm}$ lengths of flexible tygon PVC tubing (1/16" ID, 1/8" OD) to the syringes. We connected the loose ends into the appropriate input ports in the device. We

also inserted a $15 \mathrm{~cm}$ length of the tygon PVC tubing into the output port. We primed the 
device by running the syringe pump at high rates of speed $(4 \mathrm{~mL} / \mathrm{min})$ until fluid in the tubing reaches the inlet channels of the device.

iii.) Generation of Monodisperse Double Emulsions

We focused the microscope on a region of the device where the $10 \mu \mathrm{m}$ wide channel converged with the $50 \mu \mathrm{m}$ channel and contained the $50 \mu \mathrm{m}$ by $50 \mu \mathrm{m}$ orifice. We set the syringe pumps to $500 \mu \mathrm{l} / \mathrm{hr}$ for the inner phase and $500 \mu \mathrm{L} / \mathrm{hr}$ for the middle phase. We allowed continuous flow for 5 minutes to reach steady state. We ran the syringe pump at $2000 \mu \mathrm{L} / \mathrm{hr}$ for the continuous (outer) phase, also allowing 5 minutes to reach steady state. We maintained flow rates of the inner and middle phase and incrementally increased the flow at $200 \mu \mathrm{L} / \mathrm{hr}$ each minute for the continuous phase until 5,000 $\mu \mathrm{L} / \mathrm{hr}$ was reached. We allowed steady state to be reached for the continuous double emulsion generation and then acquired videos and images as double emulsions were generated in the device.

iv.) Emulsion imaging

We pipetted $50 \mu \mathrm{L}$ of solution from the bottom of the collecting vial, then placed the solution in $1 \mathrm{~mL}$ of phosphate-buffered saline (PBS). We ensured that the solution was well-mixed, then pipetted $5 \mu \mathrm{L}$ of PBS solution mixture onto a microscope slide for imaging under a fluorescent microscope. We adjusted the optical filter to the appropriate setting to ensure detection of the double emulsion encapsulated payload. We placed a scale bar on the image to determine droplet size. ImageJ was used to analyze the diameter of the double emulsion. 
D. Results and Discussion

PDMS microfluidic

devices are capable of generating water/oil/water (w/o/w) double emulsions using coaxial flow. In order to have laminar flow, it is essential that the channels are aligned properly. When the channels were properly aligned and no clogging of the channels

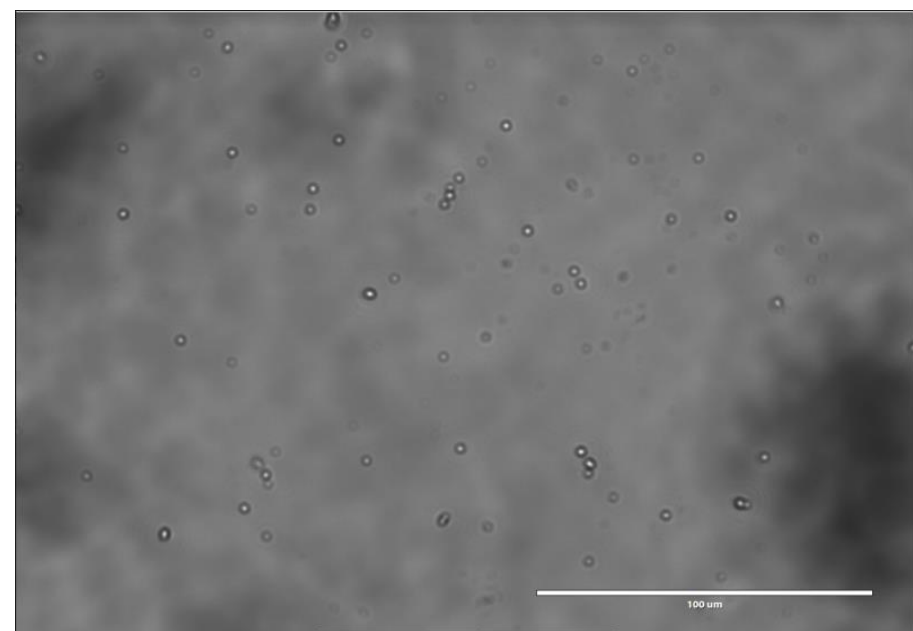

Figure 14- shows the size of double emulsions (scale bar $=100 \mu \mathrm{m})$ and monodispersity of double emulsions.

occurred, double emulsions were able to be generated as small as $1 \mu \mathrm{m}$ and ranged from 1 $\mu \mathrm{m}$ to $2 \mu \mathrm{m}$ in size (fig. 14).

We found that the current design state of the microfluidic device has many limitations. The system is very sensitive to changes in pressure and takes a significant amount of time to reach steady state (fig. 15). An introduction of

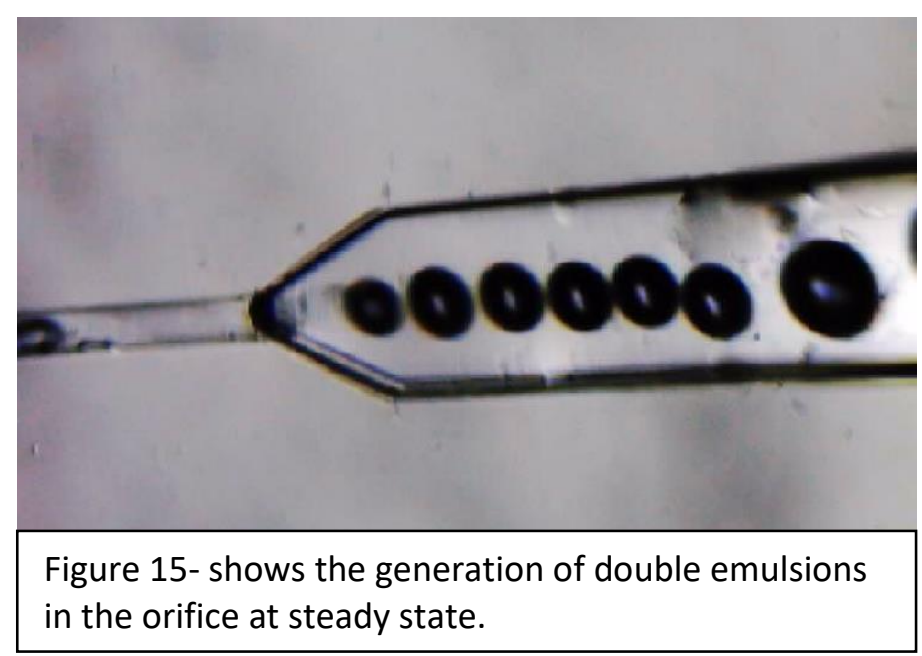
an air bubble to the system will cause the device to stop producing double emulsions for a period of time due to the variability in pressure. Another limitation is the amount of time that it takes for the microfluidic device to create enough double emulsions for treatment. It is estimated that a 
typical clinical dose requires about 1 billion double emulsions. With the current flow rate, it takes approximately 19 hours just to produce a single dose.

Changes in design can potentially address these issues. A pressure regulator can be added to maintain steady pressure, allowing a constant production of double emulsions. Designing a new microfluidic system with multiple outputs can increase the droplet production rate significantly. Both of these design changes could address the current limitations compared to other synthesis methodologies.

\section{Conclusions}

Static testing of double emulsions is an ineffective method to determine the potential effect that double emulsions may have clinically since this setup does not replicate human physiological conditions. With the current experimental methodology, double emulsions interact with the cells of interest for much longer than they would under physiological conditions. With static culture, it is difficult to test the potential use of a targeting moiety since double emulsions have a higher density than the cell culture solution, allowing them to sink and interact with the cells of interest even without a targeting moiety. With current protocols for treatment of cancer cells in static conditions, ultrasound could appear to have a negative effect on delivery of molecular compounds. Since ultrasound treatment can cause transient pores in the cell membranes, this can allow 
molecular compounds in the cell cytosol to leak outwards after passive uptake occurs. However, the fact that ultrasound can induce transient pores with double emulsions can still be viewed as a positive factor, since dynamic conditions will occur in the body which may prevent passive uptake of double emulsions by cancer cells. Thus, it is critical to have an ability to deliver the payload with spatial and temporal control.

Surfactants play a fundamental role in the stabilization and release profile of double emulsions. For storage and clinical applications it is fundamental that the double emulsion has a surfactant that is biocompatible and causes the emulsion to have a slow passive release profile. Most research pertaining to the formulation of ultrasound-responsive double emulsions has used Poloxamer 188. However we have exhibited the importance of testing other surfactants due to the release profile associated with Poloxamer 188. Double emulsions with N562 as the surfactant showed a slower release profile than Poloxamer 188.

Development of a reliable microfluidic device design is fundamental for the project moving forward. With the current design, double emulsions can be generated as small as 1 $\mu \mathrm{m}$ in size. This would allow the emulsions to travel through the capillaries if used clinically. The microfluidic device design is currently limited by slow and inconsistent production of double emulsions. To address both of these limitations, multiple inlet and multiple outlets can be incorporated into the design and the flow can be regulated by pressure instead of flow rate.

If successful, tumor-targeted double emulsions that are ultrasound-responsive could have a significant clinical impact. The drug delivery system has the ability to target the cells of interest and can use many different types of aptamers and antibodies. Along 
with that, the drug delivery system has the ability to carry many different molecular compounds, not just chemotherapy. Development of drug delivery systems that are environmentally sensitive have gained much interest since cancer cells differ in their localized environment, such as temperature and $\mathrm{pH}$. These technologies lack in the ability to cause transient pores in the cell membrane allowing for effective delivery of molecular compound to the cytosol. Therefore, ultrasound-responsive formulations could lead to more effective delivery of therapeutic molecular compounds for treatment of cancer cells.

\section{Future Work}

Being at the initial stages of development for a platform technology, a significant amount of issues have to be addressed in order for the project to progress forward. If these recommendations are met in the future, we believe that this could have a potential significant impact clinically. Below are a few topics that need to be addressed or require further investigation.

For this project to advance forward, synthesis of double emulsions should be performed by a microfluidic device. Microfluidics is currently hampered by its inability to create ample amounts of double emulsions in short period of time, and the inability for the device to maintain a constant pressure for long periods of time. To address the need to create double emulsions in a short period of time, a new design should be created that combines multiple parallel devices into one system to increase production rates. To address 
the issue for maintaining constant pressure in the device, the system input pressure should be carefully regulated instead of maintaining a constant flow rate. This will prevent small air bubbles in the system from having a significant impact on double emulsion droplet production.

Further testing of surfactants should occur to test the effect that each surfactant has on the stability of the particle. The double emulsions may have to circulate throughout the body for multiple days depending on the conjugated targeting group. In order for it to be used clinically, it must be able to withstand $37{ }^{\circ} \mathrm{C}$ without passively releasing a significant amount of the compound.

Finally, an experimental flow model should be implemented and tested. With static culture experiments, many limitations exist and this setup does not give an accurate representation of the potential impact that a targeting moiety and ultrasound can have on delivery of molecular compounds in a clinical setting. Once the implementation of a flow model occurs, the testing protocol should be extensively considered to ensure it replicates similar conditions that may be seen in vivo and the model may clearly show the effect that a targeting group and ultrasound can have on delivery of molecular compounds to cancer cells.

Current chemotherapy delivery techniques have many limitations making drug delivery devices an area of interest. If successful results are seen, ultrasound-activated double emulsions for targeted therapy could have significant impact by reducing negative systemic effects and increasing uptake by cancer cells, including dormant cancer cells. 


\section{REFERENCES}

1. Tannock, I., Cell kinetics and chemotherapy: a critical review. Cancer Treat Rep, 1978. 62(8): p. 1117-33.

2. Iwamoto, T., Clinical application of drug delivery systems in cancer chemotherapy: review of the efficacy and side effects of approved drugs. Biol Pharm Bull, 2013. 36(5): p. 715-8.

3. Tiwari, G., et al., Drug delivery systems: An updated review. Int J Pharm Investig, 2012. 2(1): p. 2-11.

4. Lakhin, A.V., V.Z. Tarantul, and L.V. Gening, Aptamers: problems, solutions and prospects. Acta Naturae, 2013. 5(4): p. 34-43.

5. Maeda, H., T. Sawa, and T. Konno, Mechanism of tumor-targeted delivery of macromolecular drugs, including the EPR effect in solid tumor and clinical overview of the prototype polymeric drug SMANCS. J Control Release, 2001. 74(13): p. 47-61.

6. Maeda, H., H. Nakamura, and J. Fang, The EPR effect for macromolecular drug delivery to solid tumors: Improvement of tumor uptake, lowering of systemic toxicity, and distinct tumor imaging in vivo. Adv Drug Deliv Rev, 2013. 65(1): p. 71-9.

7. Maeda, H., Vascular permeability in cancer and infection as related to macromolecular drug delivery, with emphasis on the EPR effect for tumor-selective drug targeting. Proc Jpn Acad Ser B Phys Biol Sci, 2012. 88(3): p. 53-71.

8. Pitt, W.G., G.A. Husseini, and B.J. Staples, Ultrasonic drug delivery--a general review. Expert Opin Drug Deliv, 2004. 1(1): p. 37-56. 
9. Schoellhammer, C.M., et al., Ultrasound-mediated gastrointestinal drug delivery. Sci Transl Med, 2015. 7(310): p. 310ra168.

10. Bekeredjian, R., et al., Ultrasound targeted microbubble destruction increases capillary permeability in hepatomas. Ultrasound Med Biol, 2007. 33(10): p. 15928.

11. Garti, N., Progress in Stabilization and Transport Phenomena of Double Emulsions in Food Applications. LWT - Food Sci Tech, 1997. 30(3): p. 222-235.

12. Chavez-Paez, M., et al., Coalescence in double emulsions. Langmuir, 2012. 28(14): p. 5934-9.

13. Holtze, C., et al., Biocompatible surfactants for water-in-fluorocarbon emulsions. Lab Chip, 2008. 8(10): p. 1632-9.

14. Datta, S.S., et al., 25th anniversary article: double emulsion templated solid microcapsules: mechanics and controlled release. Adv Mater, 2014. 26(14): p. 2205-18.

15. Chiu, Y.L., et al., Synthesis of fluorosurfactants for emulsion-based biological applications. ACS Nano, 2014. 8(4): p. 3913-20.

16. Duncanson, W.J., et al., Microfluidic fabrication of perfluorohexane-shelled double emulsions for controlled loading and acoustic-triggered release of hydrophilic agents. Langmuir, 2014. 30(46): p. 13765-70.

17. Fabiilli, M.L., et al., Delivery of water-soluble drugs using acoustically triggered perfluorocarbon double emulsions. Pharm Res, 2010. 27(12): p. 2753-65.

18. Fabiilli, M.L., et al., Assessment of the biodistribution of an [(18) F]FDG-loaded perfluorocarbon double emulsion using dynamic micro-PET in rats. Contrast Media Mol Imaging, 2013. 8(4): p. 366-74.

19. Shpak, O., et al., Ultrafast dynamics of the acoustic vaporization of phase-change microdroplets. J Acoust Soc Am, 2013. 134(2): p. 1610-21.

20. Park, J., et al., The kinetics of blood brain barrier permeability and targeted doxorubicin delivery into brain induced by focused ultrasound. J Control Release, 2012. 162(1): p. 134-42.

21. Fan, Z., et al., Spatiotemporally controlled single cell sonoporation. Proc Natl Acad Sci U S A, 2012. 109(41): p. 16486-91.

22. Kooiman, K., et al., Sonoporation of endothelial cells by vibrating targeted microbubbles. J Control Release, 2011. 154(1): p. 35-41.

23. Brujan, E.-A., Jets from pulsed-ultrasound-induced cavitation bubbles near a rigid boundary. J. Phys. D: Appl. Phys, 2017. 50(21): p. 215302.

24. Rosenberg, J.E., et al., A phase II trial of AS1411 (a novel nucleolin-targeted DNA aptamer) in metastatic renal cell carcinoma. Invest New Drugs, 2014. 32(1): p. 178-87.

25. Soundararajan, S., et al., Plasma membrane nucleolin is a receptor for the anticancer aptamer AS1411 in MV4-11 leukemia cells. Mol Pharmacol, 2009. 76(5): p. 984-91.

26. Bates, P.J., et al., G-quadruplex oligonucleotide AS1411 as a cancer-targeting agent: Uses and mechanisms. Biochim Biophys Acta, 2017. 1861(5 Pt B): p. 14141428.

27. Chen, Z. and X. Xu, Roles of nucleolin. Focus on cancer and anti-cancer therapy. Saudi Med J, 2016. 37(12): p. 1312-1318. 
28. Wise, J.F., et al., Nucleolin inhibits Fas ligand binding and suppresses Fasmediated apoptosis in vivo via a surface nucleolin-Fas complex. Blood, 2013. 121(23): p. 4729-39.

29. Farin, K., et al., Oncogenic synergism between ErbB1, nucleolin, and mutant Ras. Cancer Res, 2011. 71(6): p. 2140-51.

30. Reyes-Reyes, E.M., Y. Teng, and P.J. Bates, A new paradigm for aptamer therapeutic AS1411 action: uptake by macropinocytosis and its stimulation by a nucleolin-dependent mechanism. Cancer Res, 2010. 70(21): p. 8617-29.

31. Cole, R.H., T.M. Tran, and A.R. Abate, Double Emulsion Generation Using a Polydimethylsiloxane (PDMS) Co-axial Flow Focus Device. J Vis Exp, 2015(106): p. e53516. 